\title{
N=4, d=1 Supersymmetric Hyper-Kähler Sigma Models with Isospin Variables
}

\author{
Stefano Bellucci ${ }^{a}$, Sergey Krivonos ${ }^{b}$ and Anton Sutulin ${ }^{b}$ \\ ${ }^{a}$ INFN-Laboratori Nazionali di Frascati, Via E. Fermi 40, 00044 Frascati, Italy \\ ${ }^{b}$ Bogoliubov Laboratory of Theoretical Physics, JINR, 141980 Dubna, Russia
}

\begin{abstract}
We provide a Lagrangian formulation of $\mathcal{N}=4$ supersymmetric mechanics describing the motion of an isospin carrying particle on conformal to hyper-Kähler spaces in a non-Abelian background gauge field. In two examples we discuss in details, this background field is identified with the field of BPST instantons in the flat and Taub-NUT spaces.
\end{abstract}




\section{Introduction}

The $\mathcal{N}=4$ supersymmetric mechanics provide a nice framework for the study of many interesting features of higher dimensional theories. At the same time, the existence of a variety of off-shell $\mathcal{N}=4$ irreducible linear supermultiplets in $d=1$ [1, 2, 3, 4, 5] makes the situation in one dimension even more interesting, and just this prompted us to investigate such supersymmetric models themselves, without reference to higher dimensional counterparts. Being a supersymmetric invariant theory, the $\mathcal{N}=4$ mechanics admits a natural formulation in terms of superfields living in a standard and/or in a harmonic superspace [6], adapted to one dimension [7]. In any case the preferable approach to discuss supersymmetric mechanics is the Lagrangian one.

Being quite useful, the Lagrangian approach has one subtle point, when we try to describe the system in an arbitrary gauge background. Indeed, when the bosonic metric is conformally flat and the gauge field $\mathcal{A}_{\mu}$ is Abelian, the corresponding (bosonic) Lagrangian can be immediately written as

$$
L \sim f(x) \dot{x}_{\mu} \dot{x}_{\mu}+\mathcal{A}_{\mu}(x) \dot{x}_{\mu}+\ldots
$$

This Lagrangian can be properly extended by fermionic fields to enjoy $\mathcal{N}=4$ supersymmetry. It admits a nice superfield formulation [7, 8.

If instead the gauge field is non-Abelian (matrix-valued), then, in order to write the corresponding Lagrangian, one has to introduce extra variables $\left(\omega^{i}, \bar{\omega}_{i}\right)$ in the fundamental representation of $S U(n)$ [ . For the simplest case of the $S U(2)$ group the corresponding (bosonic) Lagrangian reads

$$
L \sim f(x) \dot{x}_{\mu} \dot{x}_{\mu}+\mathcal{A}_{\mu}^{a} I^{a} \dot{x}_{\mu}+\frac{\mathrm{i}}{2}\left(\dot{\omega}^{i} \bar{\omega}_{i}-\omega^{i} \dot{\bar{\omega}}_{i}\right),
$$

where

$$
I^{a} \sim i \omega^{i}\left(\sigma^{a}\right)_{i}^{j} \bar{\omega}_{j} .
$$

Due to the presence of only first order time derivatives in a very specific kinetic term for the isospin variables $\left(\omega^{i}, \bar{\omega}_{i}\right)$ the latter are "semi-dynamical" ones and become purely internal degrees of freedom after quantization.

The Lagrangian of the discussed type (1.2) can be also made $\mathcal{N}=4$ supersymmetric by adding the corresponding fermions $[8,9,10,11,12,13,14,15,16,17$. Moreover, it turns out that for constructing the off-shell supersymmetric Lagrangian one has to include in the game some additional auxiliary fermions which form the off-shell $\mathcal{N}=4$ supermultiplet with bosonic isospin variables $\left(\omega^{i}, \bar{\omega}_{i}\right)$. Thus, in order to deal with $\mathcal{N}=4$ supersymmetric mechanics describing the motion of the particle in some non-Abelian gauge background fields, one has to consider the coupled system of "matter" superfields $\left(x_{\mu}\right.$ and corresponding fermions) and the auxiliary superfield which contains isospin variables $\left(\omega^{i}, \bar{\omega}_{i}\right)$ together with auxiliary fermions.

There are different approaches to introduce such auxiliary superfields and couplings with them, but up to now all constructed models have been restricted to have conformally flat sigma models in the bosonic sector. This restriction has an evident source - it has been known for a long time that all linear $\mathcal{N}=4$ supermultiplets can be obtained through a dualization procedure from the $\mathcal{N}=4$ "root" supermultiplet $-\mathcal{N}=4$ hypermultiplet [18, 19, 20, 21, 22, 23], while the bosonic part of the general hypermultiplet action is always conformally flat. The only way to escape this flatness situation is to use nonlinear supermultiplets [24, 25, 26], instead of linear ones.

The main aim of the present paper is to construct the Lagrangian formulation of $\mathcal{N}=4$ supersymmetric mechanics on hyper-Kähler spaces in the non-Abelian background gauge fields. To achieve this goal we will perform two steps

- First, we introduce the coupling of the "matter" $\mathcal{N}=4$ tensor supermultiplet $\mathcal{V}^{i j}$ with an auxiliary fermionic supermultiplet $\Psi^{\hat{\alpha}}$ in a such way to have the proper action for matter fields and isospin variables (1.2). This procedure was developed in [13. On this step we still have conformally flat three-dimensional sigma-model in the bosonic sector.

- As the next step, following [24, we dualize the auxiliary component $A$ of the tensor supermultiplet into a fourth physical boson, finishing with the action having the hyper-Kähler sigma-model in the bosonic sector. 
The resulting action contains a wide class of $\mathcal{N}=4$ supersymmetric mechanics describing the motion of an isospin-carrying particle over spaces with non-trivial hyper-Kähler geometry and in the presence of a non-Abelian background gauge field. In two examples we discussed in details, these backgrounds correspond to the field of the BPST instanton in the flat and Taub-NUT spaces.

\section{$2 \mathcal{N}=4$ supersymmetric isospin particles in conformally flat spaces}

One of the possible ways to incorporate the isospin-like variables in the Lagrangian of supersymmetric mechanics is to couple the basic superfields with auxiliary fermionic superfields $\Psi^{\hat{\alpha}}, \bar{\Psi}_{\hat{\alpha}}$, which contain these isospin variables 13. Such a coupling, being written in a standard $\mathcal{N}=4$ superspace, has to be rather special, in order to provide a kinetic term of the first order in time derivatives for the isospin variables and to describe the auxiliary fermionic components present in $\Psi^{\hat{\alpha}}, \bar{\Psi}_{\hat{\alpha}}$. Following $[13$, we introduce the coupling of auxiliary $\Psi$ superfields with some arbitrary, for the time being, $\mathcal{N}=4$ supermultiplet $X$ as

$$
S_{c}=-\frac{1}{32} \int d t d^{4} \theta(X+g) \Psi^{\hat{\alpha}} \bar{\Psi}_{\hat{\alpha}}, \quad g=\text { const } .
$$

The $\Psi$ supermultiplet is subjected to the irreducible conditions [5]

$$
D^{i} \Psi^{1}=0, \quad D^{i} \Psi^{2}+\bar{D}^{i} \Psi^{1}=0, \quad \bar{D}_{i} \Psi^{2}=0,
$$

and thus it contains four fermionic and four bosonic components

$$
\psi^{\hat{\alpha}}=\Psi^{\hat{\alpha}}\left|, \quad u^{i}=-D^{i} \bar{\Psi}^{2}\right|, \quad \bar{u}_{i}=\bar{D}_{i} \Psi^{1} \mid,
$$

where the symbol $\mid$ denotes the $\theta=\bar{\theta}=0$ limit and $\mathcal{N}=4$ covariant derivatives obey standard relations

$$
\left\{D^{i}, \bar{D}_{j}\right\}=2 \mathrm{i} \delta_{j}^{i} \partial_{t} .
$$

It has been demonstrated in [13] that if the $\mathcal{N}=4$ superfield $X$ is subjected to the constraints [5, 27]

$$
D^{i} D_{i} X=0, \quad \bar{D}_{i} \bar{D}^{i} X=0, \quad\left[D^{i}, \bar{D}_{i}\right] X=0,
$$

then the component action which follows from (2.1) can be written as

$$
\begin{aligned}
S_{c} & =\int d t\left[-(x+g)\left(\rho^{1} \bar{\rho}^{2}-\rho^{2} \bar{\rho}^{1}\right)-\frac{i}{4}(x+g)\left(\dot{u}^{i} \bar{u}_{i}-u^{i} \dot{\bar{u}}_{i}\right)+\frac{1}{4} A_{i j} u^{i} \bar{u}^{j}\right. \\
& \left.+\frac{1}{2} \eta_{i}\left(\bar{u}^{i} \bar{\rho}^{2}+u^{i} \rho^{2}\right)+\frac{1}{2} \bar{\eta}^{i}\left(u_{i} \rho^{1}+\bar{u}_{i} \bar{\rho}^{1}\right)\right]
\end{aligned}
$$

where the new fermionic components $\rho^{\hat{\alpha}}, \bar{\rho}_{\hat{\alpha}}$ are defined as

$$
\rho^{\hat{\alpha}}=\dot{\psi}^{\hat{\alpha}}, \quad \bar{\rho}_{\hat{\alpha}}=\dot{\bar{\psi}}_{\hat{\alpha}} .
$$

The components of the superfield $X$ entering the action (2.6) have been introduced as

$$
x=X\left|, \quad A_{i j}=A_{(i j)}=\frac{1}{2}\left[D_{i}, \bar{D}_{j}\right] X\right|, \quad \eta^{i}=-i D^{i} X\left|, \quad \bar{\eta}_{i}=-i \bar{D}_{i} X\right| .
$$

What makes the action (2.6) interesting is that, despite the non-local definition of the fermionic components $\rho^{\hat{\alpha}}, \bar{\rho}_{\hat{\alpha}}$ (2.7), the action is invariant under the following $\mathcal{N}=4$ supersymmetry transformations:

$$
\begin{aligned}
& \delta \rho^{1}=-\bar{\epsilon}^{i} \dot{\bar{u}}_{i}, \quad \delta \rho^{2}=\epsilon_{i} \dot{\bar{u}}^{i}, \quad \delta u^{i}=-2 i \epsilon^{i} \bar{\rho}^{1}+2 i \bar{\epsilon}^{i} \bar{\rho}^{2}, \quad \delta \bar{u}_{i}=-2 i \epsilon_{i} \rho^{1}+2 i \bar{\epsilon}_{i} \rho^{2} \\
& \delta x=-i \epsilon_{i} \eta^{i}-i \bar{\epsilon}^{i} \bar{\eta}_{i}, \quad \delta \eta^{i}=-\bar{\epsilon}^{i} \dot{x}-i \bar{\epsilon}^{j} A_{j}^{i}, \quad \delta \bar{\eta}_{i}=-\epsilon_{i} \dot{x}+i \epsilon_{j} A_{i}^{j}, \quad \delta A_{i j}=-\epsilon_{(i} \dot{\eta}_{j)}+\bar{\epsilon}_{(i} \dot{\bar{\eta}}_{j)} .
\end{aligned}
$$

In the action (2.6) the fermionic fields $\rho^{\hat{\alpha}}, \bar{\rho}_{\hat{\alpha}}$ are auxiliary ones, and thus they can be eliminated by their equations of motion

$$
\rho^{1}=\frac{1}{2(x+g)} \eta_{i} \bar{u}^{i}, \quad \rho^{2}=-\frac{1}{2(x+g)} \bar{\eta}^{i} \bar{u}_{i} .
$$


Finally, the action describing the interaction of $\Psi$ and $X$ supermultiplets acquires a very simple form

$$
S_{c}=\frac{1}{4} \int d t\left[-i(x+g)\left(\dot{u}^{i} \bar{u}_{i}-u^{i} \dot{\bar{u}}_{i}\right)+A_{i j} u^{i} \bar{u}^{j}+\frac{1}{x+g} \eta_{i} \bar{\eta}_{j}\left(u^{i} \bar{u}^{j}+u^{j} \bar{u}^{i}\right)\right] .
$$

Thus, in the fermionic superfields $\Psi$ only the bosonic components $u^{i}, \bar{u}_{i}$, entering the action with a kinetic term linear in time-derivatives, survive.

Comparing our action (2.11) with (1.2) one may see that the term describing the interaction with the gauge field is just (one has firstly to rescale isospin variables to bring their kinetic term to the flat form (1.2)

$$
\frac{1}{g+x} A_{i j} \omega^{i} \bar{\omega}^{j}, \quad \omega^{i}=\sqrt{g+x} u^{i}
$$

It is clear that the explicit form of the gauge field could be read off only after expressing the (for the time being) "auxiliary" components $A_{i j}$ in terms of physical bosons present in the model. Thus, in order to be meaningful, the action (2.1) has to be extended by the action for the supermultiplet $X$ itself:

$$
S=S_{x}+S_{c}=-\frac{1}{32} \int d t d^{4} \theta \mathcal{F}(X)+S_{c},
$$

where $\mathcal{F}(X)$ is an arbitrary function depending on superfield $X$.

If the superfield $X$ obeying (2.5) is considered as an independent superfield, then the components $A_{i j}(2.8)$ are auxiliary ones, and they have to be eliminated by their equations of motion. The resulting action describes $\mathcal{N}=4$ supersymmetric mechanics with one physical boson $x$ and four physical fermions $\eta^{i}, \bar{\eta}_{j}$ interacting with isospin variables $u^{i}, \bar{u}_{i}$. Just this system has been considered in [10, 12, 13.

It is clear that treating the scalar bosonic superfield $X$ as an independent one is too restrictive, because the constraints (2.5) leave in this supermultiplet only one physical bosonic component $x$, which is not enough to describe the isospin particle. In the present approach, the way to overcome this limitation was proposed in [15, 17. The key point is to treat the superfield $X$ as a composite one, constructed from $\mathcal{N}=4$ supermultiplets with a larger number of physical bosons. The reasonable superfield from which it is possible to construct the superfield $X$ is the $\mathcal{N}=4$ tensor supermultiplet $\mathcal{V}^{i j}[28,29$.

\section{Tensor supermultiplet}

The $\mathcal{N}=4$ tensor supermultiplet is described by the triplet of bosonic $\mathcal{N}=4$ superfields $\mathcal{V}^{i j}=\mathcal{V}^{i j}$ subjected to the constraints

$$
D^{(i} \mathcal{V}^{j k)}=\bar{D}^{(i} \mathcal{V}^{j k)}=0, \quad\left(\mathcal{V}^{i j}\right)^{\dagger}=\mathcal{V}_{i j},
$$

which leave in $\mathcal{V}^{i j}$ the following independent components:

$$
v^{a}=-\frac{\mathrm{i}}{2}\left(\sigma^{a}\right)_{i}{ }^{j} \mathcal{V}_{j}^{i}\left|, \quad \lambda^{i}=\frac{1}{3} D^{j} \mathcal{V}_{j}^{i}\right|, \quad \bar{\lambda}_{i}=\frac{1}{3} \bar{D}_{j} \mathcal{V}_{i}^{j}\left|, \quad A=\frac{\mathrm{i}}{6} D^{i} \bar{D}^{j} \mathcal{V}_{i j}\right| .
$$

Thus, its off-shell component field content is $(3,4,1)$, i.e. three physical $v^{a}$ and one auxiliary $A$ bosons and four fermions $\lambda^{i}, \bar{\lambda}_{i}[28,29]$. Under $\mathcal{N}=4$ supersymmetry these components transform as follows:

$$
\begin{aligned}
& \delta v^{a}=\mathrm{i} \epsilon^{i}\left(\sigma^{a}\right)_{i}^{j} \bar{\lambda}_{j}-\mathrm{i} \lambda^{i}\left(\sigma^{a}\right)_{i}^{j} \bar{\epsilon}_{j}, \quad \delta A=\bar{\epsilon}_{i} \dot{\lambda}^{i}-\epsilon^{i} \dot{\bar{\lambda}}_{i}, \\
& \delta \lambda^{i}=\mathrm{i} \epsilon^{i} A+\epsilon^{j}\left(\sigma^{a}\right)_{j}^{i} \dot{v}^{a}, \quad \delta \bar{\lambda}_{i}=-\mathrm{i} \bar{\epsilon}_{i} A+\left(\sigma^{a}\right)_{i}^{j} \bar{\epsilon}_{j} \dot{v}^{a} .
\end{aligned}
$$

Now one may check that the composite superfield

$$
X=\frac{1}{|\mathcal{V}|} \equiv \frac{1}{\sqrt{\mathcal{V}^{a} \mathcal{V}^{a}}}
$$

where $\mathcal{V}^{a}=-\frac{\mathrm{i}}{2}\left(\sigma^{a}\right)_{i}{ }^{j} \mathcal{V}_{j}^{i}$, obeys (2.5) in virtue of (2.14). Clearly, now all components of the $X$ super-

field, i.e. the physical boson $x$, fermions $\eta^{i}, \bar{\eta}_{i}$ and auxiliary fields $A^{i j}$ (2.8) are expressed through the components of the $\mathcal{V}^{i j}$ supermultiplet (2.15) as

$$
\begin{aligned}
& x=\frac{1}{|v|}, \quad \eta^{i}=\frac{v^{a}}{|v|^{3}}\left(\lambda \sigma^{a}\right)^{i}, \quad \bar{\eta}_{i}=\frac{v^{a}}{|v|^{3}}\left(\sigma^{a} \bar{\lambda}\right)_{i}, \\
& A_{j}^{i}=-3 \frac{v^{a} v^{b}}{|v|^{5}}\left(\lambda \sigma^{a}\right)^{i}\left(\sigma^{b} \bar{\lambda}\right)_{j}-\frac{v^{a}\left(\sigma^{a}\right)_{j}^{i}}{|v|^{3}} A+\frac{1}{|v|^{3}} \epsilon^{a b c} v^{a} \dot{v}^{b}\left(\sigma^{c}\right)_{j}^{i}+\frac{1}{|v|^{3}}\left(\delta_{j}^{i} \lambda^{k} \bar{\lambda}_{k}-\lambda_{j} \bar{\lambda}^{i}\right) .
\end{aligned}
$$


The third term in the above expression for $A^{i j}$ being substituted in the action (2.11) provides us with the term

$$
\mathcal{A}_{b}^{a} I^{a} \dot{v}^{b}=\frac{1}{\left(g+\frac{1}{|v|}\right)|v|^{3}} \epsilon^{c a b} v^{c} I^{a} \dot{v}^{b}, \quad I^{c}=\frac{1}{2} \omega^{i}\left(\sigma^{c}\right)_{i}^{j} \bar{\omega}_{j}, \omega^{i}=\sqrt{g+\frac{1}{|v|}} u^{i}
$$

Thus, for the $g=0$ case our gauge field describes the magnetic field of a Wu-Yang monopole [30].

Finally, one should note that, while dealing with the tensor supermultiplet $\mathcal{V}^{i j}$, one may generalize the $S_{x}$ action (2.13) to have the full action in the form

$$
S=S_{v}+S_{c}=-\frac{1}{32} \int d t d^{4} \theta \mathcal{F}(\mathcal{V})+S_{c}
$$

where $\mathcal{F}(\mathcal{V})$ is now an arbitrary function of $\mathcal{V}^{i j}$. After eliminating the auxiliary component $A$ in the component form of (2.20) we will obtain the action describing the $\mathcal{N}=4$ supersymmetric three-dimensional isospin particle moving in the magnetic field of a Wu-Yang monopole and in some specific scalar potential [15] 1 .

To close this Section one should mention that, while dealing with the tensor supermultiplet $\mathcal{V}^{i j}$, the structure of the action $S_{c}$ (2.1) can be further generalized to be [17]

$$
S_{c}=-\frac{1}{32} \int d t d^{4} \theta Y \Psi^{\hat{\alpha}} \bar{\Psi}_{\hat{\alpha}}
$$

with $Y$ obeying

$$
\frac{\partial^{2}}{\partial v_{a} \partial v_{a}} Y=0
$$

Clearly, our choice $Y=\frac{1}{|v|}+g$ corresponds to spherically-symmetric solutions of (2.22).

\section{Hyper-Kähler sigma model with isospin variables}

One of the most attractive features of our approach is the unified structure of the action $S_{c}$ (2.1) which has the same form for any type of supermultiplets, which we are using to construct a composite superfield $X$. Just this opens the way to relate the different systems via duality transformations. Indeed, it has been known for a long time [1, 2, 3, 4, 22, 23] that in one dimension one may switch between supermultiplets with a different number of physical bosons, by expressing the auxiliary components through the time derivative of physical bosons, and vice versa. Here we will use this mechanism to obtain the action with four physical bosonic components starting from the action for the tensor multiplet (2.20). In what follows, to make some expressions more transparent, we will use, sometimes, the following stereographic coordinates for the bosonic components of tensor supermultiplet (2.15):

$$
V^{11}=2 \mathrm{i} \frac{e^{u}}{1+\Lambda \bar{\Lambda}} \Lambda, \quad V^{22}=-2 \mathrm{i} \frac{e^{u}}{1+\Lambda \bar{\Lambda}} \bar{\Lambda}, \quad V^{12}=-\mathrm{i} e^{u}\left(\frac{1-\Lambda \bar{\Lambda}}{1+\Lambda \bar{\Lambda}}\right) .
$$

The crucial step in the dualization of the last auxiliary field $A$ (2.18) into a fourth physical boson is the transformation property of the following combination:

$$
\hat{A}=B_{a} \dot{v}_{a}-f_{, a}\left(\lambda \sigma^{a} \bar{\lambda}\right)+f A, \quad f_{, a} \equiv \frac{\partial}{\partial v_{a}}
$$

where

$$
f=\frac{1}{|v|}, \quad \text { and }, \quad B_{1}=-\frac{v_{2}\left(v_{3}+|v|\right)}{\left(v_{1}^{2}+v_{2}^{2}\right)|v|}, \quad B_{2}=\frac{v_{1}\left(v_{3}+|v|\right)}{\left(v_{1}^{2}+v_{2}^{2}\right)|v|}, \quad B_{3}=0 .
$$

Indeed, one may easily check that $\hat{A}$ transforms as a full time derivative under $\mathcal{N}=4$ supersymmetry. Therefore, in accordance with the general idea of the dualization procedure one may replace $\hat{A}$ by a new physical bosonic field $\phi$ as

$$
\hat{A}=\dot{\phi} .
$$

\footnotetext{
${ }^{1}$ An alternative description of the same system has been recently constructed in [16]
} 
What is much more interesting is that the choice for $f$ and $B_{a}$ in (3.3) is not unique. It has been proved in 24 that $\hat{A}(3.2)$ transforms as a full time derivative, if the functions $f$ and $B_{a}$ satisfy the equations

$$
\triangle_{3} f \equiv f_{, a a}=0, \quad f_{, a}=\epsilon_{a b c} B_{c, b} .
$$

Thus, one may construct a more general action for four-dimensional $\mathcal{N}=4$ supersymmetric mechanics using the component action for the tensor supermultiplet and substituting there the new dualized version of the auxiliary component $A$ (3.2).

Integrating over theta's in (2.20) and eliminating the auxiliary fermions $\rho^{\hat{\alpha}}(2.10),(2.18)$, we will get the following component action for the tensor supermultiplet:

$$
\begin{aligned}
S= & \frac{1}{8} \int d t\left[F\left(\dot{v}_{a} \dot{v}_{a}+A^{2}\right)+\mathrm{i}\left(\dot{\xi}^{i} \bar{\xi}_{i}-\xi^{i} \dot{\bar{\xi}}_{i}\right)+\mathrm{i} \epsilon_{a b c} \frac{F_{, a}}{F} \dot{v}_{b} \Sigma_{c}-\mathrm{i} \frac{F_{, a}}{F} \Sigma_{a} A-\frac{1}{6} \frac{\triangle_{3} F}{F^{2}} \Sigma_{a} \Sigma_{a}\right. \\
& -2 \mathrm{i}\left(\dot{w}^{i} \bar{w}_{i}-w^{i} \dot{\bar{w}}_{i}\right)+4 \frac{1+3 g|v|}{F(1+g|v|)^{2}|v|^{4}}\left(v_{a} I_{a}\right)\left(v_{b} \Sigma_{b}\right)-4 \frac{g}{F(1+g|v|)^{2}|v|}\left(I_{a} \Sigma_{a}\right) \\
& \left.-\frac{4 \mathrm{i}}{(1+g|v|)|v|^{2}}\left(v_{a} I_{a}\right) A+\frac{4 \mathrm{i}}{(1+g|v|)|v|^{2}} \epsilon_{a b c} v_{a} \dot{v}_{b} I_{c}\right],
\end{aligned}
$$

where

$$
F=\triangle_{3} \mathcal{F}(\mathcal{V}) \mid, \quad I^{a}=\frac{\mathrm{i}}{2}\left(w \sigma^{a} \bar{w}\right), \quad \Sigma^{a}=-\mathrm{i}\left(\xi \sigma^{a} \bar{\xi}\right),
$$

and the re-scaled fermions and isospin variables are chosen to be

$$
\xi^{i}=\sqrt{F} \lambda^{i}, \quad w^{i}=\sqrt{g+\frac{1}{|v|}} u^{i} .
$$

Substituting (3.2) into (3.6), we obtain the resulting action

$$
\begin{aligned}
S= & \frac{1}{8} \int d t\left[F\left(\dot{v}_{a} \dot{v}_{a}+\frac{1}{f^{2}}\left(\dot{\phi}-B_{a} \dot{v}_{a}\right)^{2}\right)+\mathrm{i}\left(\dot{\xi}^{i} \bar{\xi}_{i}-\xi^{i} \dot{\bar{\xi}}_{i}\right)-2 \mathrm{i}\left(\dot{w}^{i} \bar{w}_{i}-w^{i} \dot{\bar{w}}_{i}\right)\right. \\
& -\mathrm{i}\left[\frac{1}{f} \delta_{a b}\left(\dot{\phi}-B_{c} \dot{v}_{c}\right)+\epsilon_{a b c} \dot{v}_{c}\right]\left(\frac{F_{, a}}{F} \Sigma_{b}+\frac{4}{(1+g|v|)|v|^{2}} v_{a} I_{b}\right) \\
& +\frac{4}{F} \frac{1+3 g|v|}{(1+g|v|)^{2}|v|^{4}}\left(v_{a} I_{a}\right)\left(v_{b} \Sigma_{b}\right)-\frac{1}{F} \frac{4 g}{(1+g|v|)^{2}|v|}\left(I_{a} \Sigma_{a}\right) \\
& \left.+\frac{1}{3 F^{2}}\left(\frac{F_{, a} f_{, a}}{f}-\frac{F f_{, a} f_{, a}}{f^{2}}-\frac{1}{2} \triangle_{3} F\right) \Sigma_{b} \Sigma_{b}\right] .
\end{aligned}
$$

The action (3.9) is our main result. It describes a motion of a $\mathcal{N}=4$ supersymmetric four-dimensional isospin carrying particle in the non-Abelian gauge field with potential

$$
\mathcal{A}_{\mu} \dot{x}_{\mu}=-\mathrm{i}\left[\frac{1}{f} \delta_{a b}\left(\dot{\phi}-B_{c} \dot{v}_{c}\right)+\epsilon_{a b c} \dot{v}_{c}\right] \frac{4}{(1+g|v|)|v|^{2}} v_{a} I_{b}
$$

where $x_{\mu}=\left(v_{a}, \phi\right)$. The four-dimensional bosonic metric of our model is defined in terms of two functions: the bosonic part of the pre-potential $F$ (3.7) and the harmonic function $f$ (3.5). The supersymmetric version of the coupling with the monopole (second line in the action (3.9) ) is defined by the same harmonic function $f$ and the coupling constant $g$. In the more general case (2.21), we will have two harmonic functions - $f$ and $Y$, besides the pre-potential $F$.

Among all possible systems with the action (3.9) there is a very interesting sub-class which corresponds to hyper-Kähler sigma models in the bosonic sector. This case is distinguished by the condition

$$
F=f .
$$

Clearly, in this case the bosonic kinetic term of the action (3.9) acquires the familiar form of the one dimensional version of the general Hawking-Gibbons solution for four-dimensional hyper-Kähler metrics with one triholomorphic isometry [31:

$$
S_{k i n}=\frac{1}{8} \int d t\left[f \dot{v}_{a} \dot{v}_{a}+\frac{1}{f}\left(\dot{\phi}-B_{a} \dot{v}_{a}\right)^{2}\right], \quad \triangle_{3} f=0, \operatorname{rot} \vec{B}=\vec{\nabla} f .
$$


It is worth to note that the bosonic part of $\mathcal{N}=4$ supersymmetric four dimensional sigma models in one dimension does not necessarily have to be a hyper-Kähler one. This fact is reflected in the arbitrariness of the pre-potential $F$ in the action (3.9). Only under the choice $F=f$ the bosonic kinetic term is reduced to the Gibbons-Hawking form (3.12). Let us note that for hyper-Kähler cases the four-fermionic term in the action (3.9) disappears. This fact has been previously established in [24]. Now we can see that the additional interaction with background non-Abelian gauge field does not destroy these nice properties.

Among all possible bosonic metrics one may easily find the following interesting ones.

\section{Conformally flat spaces.}

There are two choices for the function $f$ which correspond to the conformally flat metrics in the bosonic sector. Both of them with

$$
f=\frac{1}{|v|} \quad \text { and } f=\text { const }, \quad B_{a}=0
$$

give rise to supersymmetric mechanics with the hypermultiplet describing the particle moving in the field of a BPST instanton [17.

Let us remind that in both these cases we have not specified the pre-potential $F$ yet. Therefore, the full metrics in the bosonic sector is defined up to this function.

\section{Taub-NUT space.}

One should stress that the previous two cases are unique, because only for these choices of $f$ the resulting action (3.9) can be formulated in terms of linear $\mathcal{N}=4$ hypermultiplet 18, 19, 20, 21. With other solutions for $f$ we come to the theory with the nonlinear $\mathcal{N}=4$ hypermultiplet [24, 25]. Among the possible solutions for $f$ which belongs to this new situation the simplest one corresponds to one center Taub-NUT metrics with

$$
f=p_{1}+\frac{p_{2}}{|v|}, \quad p_{1}, p_{2}=\text { const. }
$$

In order to achieve the maximally symmetric case, we will chose these constants as

$$
p_{1}=g, \quad p_{2}=1 \quad \rightarrow \quad f=g+\frac{1}{|v|} .
$$

With such a definition $f$ coincides with the function $Y=g+\frac{1}{|v|}(2.21)$ entering in our basic action $S_{c}$ in (2.1), (2.17). To get the Taub-NUT metrics, one has also to fix the pre-potential $F$ to be equal to $f$. The resulting action which describes the $\mathcal{N}=4$ supersymmetric isospin carrying particle moving in a Taub-NUT space reads

$$
\begin{aligned}
S_{\text {Taub-NUT }}= & \frac{1}{8} \int d t\left[\left(g+\frac{1}{|v|}\right) \dot{v}_{a} \dot{v}_{a}+\frac{1}{\left(g+\frac{1}{|v|}\right)}\left(\dot{\phi}-B_{a} \dot{v}_{a}\right)^{2}+\mathrm{i}\left(\dot{\xi}^{i} \bar{\xi}_{i}-\xi^{i} \dot{\bar{\xi}}_{i}\right)-2 \mathrm{i}\left(\dot{w}^{i} \bar{w}_{i}-w^{i} \dot{\bar{w}}_{i}\right)\right. \\
& +\frac{\mathrm{i}}{(1+g|v|)|v|^{2}}\left[\frac{v_{a}}{\left(g+\frac{1}{|v|}\right)}\left(\dot{\phi}-B_{c} \dot{v}_{c}\right)-\epsilon_{a b c} v_{b} \dot{v}_{c}\right]\left(\Sigma_{a}-4 I_{a}\right) \\
& \left.+\frac{4(1+3 g|v|)}{(1+g|v|)^{3}|v|^{3}}\left(v_{a} I_{a}\right)\left(v_{b} \Sigma_{b}\right)-\frac{4 g}{(1+g|v|)^{3}}\left(I_{a} \Sigma_{a}\right)\right] .
\end{aligned}
$$

The bosonic term in the second line of this action can be rewritten as

$$
\mathcal{A}_{a} I_{a}=\frac{\mathrm{i}}{2}\left[\frac{1}{f} \frac{\partial \log f}{\partial v_{a}}\left(\dot{\phi}-B_{c} \dot{v}_{c}\right)-\epsilon_{a b c} \frac{\partial \log f}{\partial v_{b}} \dot{v}_{c}\right] I_{a}
$$

where $f$ is defined in (3.15). In this form the vector potential $\mathcal{A}_{a}$ coincides with the potential of a Yang-Mills $S U(2)$ instanton in the Taub-NUT space [32, 33, if we may view $I_{a}$, as defined in (3.7), as proper isospin matrices. The remaining terms in the second and third lines of (3.16) provide a $\mathcal{N}=4$ supersymmetric extension of the instanton.

Finally, to close this Section, let us note that more general non-Abelian backgrounds can be obtained from the multi-centered solutions of the equation for the harmonic function $Y$ (2.22), which defined the coupling of the tensor supermultiplet with auxiliary fermionic ones. Thus, the variety models we 
constructed are defined through three functions: pre-potential $\mathcal{F}(2.20)$ which is an arbitrary function, 3D harmonic function $Y$ (2.21), (2.22) defining the coupling with isospin variables and, through, again 3D harmonic, function $f$ (3.2), (3.5) which appeared during the dualization of the auxiliary component of the tensor supermultiplet. It is clear that we can always redefine $F$ to be $F=\tilde{F} f$. Thus, all our models are conformal to hyper-Kähler sigma models with $\mathcal{N}=4$ supersymmetry describing the motion of a particle in the background of non-Abelian field of the corresponding instantons.

\section{Conclusion}

In the present paper we constructed the Lagrangian formulation of $\mathcal{N}=4$ supersymmetric mechanics with hyper-Kähler sigma models in the bosonic sector in the non-Abelian background gauge field. The resulting action includes the wide class of $\mathcal{N}=4$ supersymmetric mechanics describing the motion of an isospin-carrying particle over spaces with non-trivial geometry. In two examples we discussed in details, the background fields are identified with the field of BPST instantons in the flat and Taub-NUT spaces.

The approach we used in the paper utilized two ideas: (i) the coupling of matter supermultiplets with an auxiliary fermionic supermultiplet $\Psi^{\hat{\alpha}}$ containing on-shell four physical fermions and four auxiliary bosons playing the role of isospin variables and (ii) the dualization of the auxiliary component $A$ of the tensor supermultiplet into a fourth physical boson. The final action we constructed contains three arbitrary functions: the pre-potential $\mathcal{F}$, a 3D harmonic function $Y$ which defines the coupling with isospin variables and, again 3D harmonic, a function $f$ which appeared during the dualization of the auxiliary component of the tensor supermultiplet. The usefulness of the proposed approach is demonstrated by the explicit example of the simplest system with non-trivial geometry - the $\mathcal{N}=4$ supersymmetric action for one-center Taub-NUT metrics. We identified the background gauge field in this case, which appears automatically in our framework, with the field of the BPST instanton in the Taub-NUT space. Thus, one may hope that the other actions will possess the same structure.

Of course, the presented results are just preliminary in the full understanding of $\mathcal{N}=4$ supersymmetric hyper-Kähler sigma models in non-Abelian backgrounds. Among interesting, still unanswered questions, one should note the following ones:

- The full analysis of the general coupling with an arbitrary harmonic function $Y$ has yet to be carried out.

- The structure of the background gauge field has to be further clarified: is this really the field of some monopole (instanton) for any hyper-Kähler metrics?

- The Hamiltonian construction is really needed. Let us note that the Supercharges have to be very specific, because the four-fermions coupling is absent in the case of HK metrics!

- It is quite interesting to check the existence of the conserved Runge-Lenz vector in the fully supersymmetric version.

- The explicit examples of other hyper-Kähler metrics (say, multi-centered Eguchi-Hanson and TaubNUT ones) would be very useful.

- The questions of quantization and analysis of the spectra, at least in the cases of well known, simplest hyper-Kähler metrics, are doubtless urgent tasks.

Finally, let us stress that our construction is restricted to the case of hyper-Kähler metrics with one translational (triholomorphic) isometry. It will be very interesting to find a similar construction applicable to the case of geometries with rotational isometry. We hope this can be done within the approach discussed in [34.

\section{Acknowledgements}

We thank Andrey Shcherbakov for useful discussions. S.K. and A.S. are grateful to the Laboratori Nazionali di Frascati for hospitality. This work was partially supported by the grants RFBF-09-02-01209 and 09-02-91349, by Volkswagen Foundation grant I/84 496 as well as by the ERC Advanced Grant no. 226455, "Supersymmetry, Quantum Gravity and Gauge Fields" (SUPERFIELDS). 


\section{References}

[1] S.J. Gates, L. Rana, Ultramultiplets: A New representation of rigid 2-d, N=8 supersymmetry, Phys. Lett. B342 (1995) 132, arXiv:hep-th/9410150

[2] A. Pashnev, F. Toppan, On the Classification of N-extended Supersymmetric Quantum Mechanical Systems, J. Math. Phys. 42 (2001) 5257, arXiv:hep-th/0010135.

[3] M. Faux, S.J. Gates, Adinkras: A Graphical technology for supersymmetric representation theory, Phys. Rev. D71 (2005) 065002, arXiv:hep-th/0408004.

[4] S. Bellucci, S.J. Gates, E. Orazi, A Journey through garden algebras, Lect. Notes Phys. 698 (2006) 1-47, arXiv:hep-th/0602259.

[5] E. Ivanov, S. Krivonos, O. Lechtenfeld, $N=4, d=1$ supermultiplets from nonlinear realizations of $D(2,1 ; \alpha)$, Class. Quant. Grav. 21 (2004) 1031, arXiv:hep-th/0310299.

[6] A.S. Galperin, E.A. Ivanov, V.I. Ogievetsky, E.S. Sokatchev, Harmonic Superspace, Cambridge, UK: Univ. Press. (2001), 306pp.

[7] E. Ivanov, O. Lechtenfeld, $N=4$ Supersymmetric Mechanics in Harmonic Superspace, JHEP 0309 (2003) 073, arXiv:hep-th/0307111.

[8] M. Konyushikhin, A. Smilga, Self-duality and supersymmetry, Phys. Lett. B689 (2010) 95, arXiv:0910.5162[hep-th].

[9] E.A. Ivanov, M.A. Konyushikhin, A.V. Smilga, SQM with non-Abelian self-dual fields: harmonic superspace description, JHEP 1005 (2010) 033, arXiv:0912.3289[hep-th].

[10] S. Fedoruk, E. Ivanov, O. Lechtenfeld, Supersymmetric Calogero models by gauging, Phys. Rev. D79 (2009) 105015, arXiv:0812.4276[hep-th].

[11] M. Gonzales, Z. Kuznetsova, A. Nersessian, F. Toppan, V. Yeghikyan, Second Hopf map and supersymmetric mechanics with Yang monopole, Phys. Rev. D80 (2009) 025022, arXiv:0902.2682[hep-th].

[12] S. Fedoruk, E. Ivanov, O. Lechtenfeld, $O S p(4 \mid 2)$ Superconformal Mechanics, JHEP 0908 (2009) 081, arXiv:0905.4951[hep-th].

[13] S. Bellucci, S. Krivonos, Potentials in $N=4$ superconformal mechanics, Phys. Rev. D80 (2009) 065022, arXiv:0905.4633[hep-th].

[14] S. Krivonos, O. Lechtenfeld, $S U(2)$ reduction in $N=4$ supersymmetric mechanics, Phys. Rev. D80 (2009) 045019, arXiv:0906.2469[hep-th].

[15] S. Bellucci, S. Krivonos, A. Sutulin,Three dimensional $N=4$ supersymmetric mechanics with WuYang monopole, Phys. Rev. D81 (2010) 105026, arXiv:0911.3257[hep-th].

[16] E. Ivanov, M. Konyushikhin, N=4, 3D Supersymmetric Quantum Mechanics in Non-Abelian Monopole Background, Phys. Rev. D 82 (2010) 085014, arXiv:1004.4597[hep-th].

[17] S. Krivonos, O. Lechtenfeld, A. Sutulin, N=4 Supersymmetry and the BPST Instanton, Phys. Rev. D81 (2010) 085021, arXiv:1001.2659[hep-th].

[18] R.A. Coles, G. Papadopoulos, The Geometry of the one-dimensional supersymmetric nonlinear sigma models, Class. Quant. Grav. 7 (1990) 427.

[19] G.W. Gibbons, G. Papadopoulos, K.S. Stelle, HKT and OKT Geometries on Soliton Black Hole Moduli Spaces, Nucl. Phys. B508 (1997) 623, arXiv:hep-th/9706207.

[20] S. Hellerman, J. Polchinski, Supersymmetric quantum mechanics from light cone quantization, in: Shifman, M.A. (ed.), The many faces of the superworld, arXiv:hep-th/9908202.

[21] C.M. Hull, The geometry of supersymmetric quantum mechanics, arXiv:hep-th/9910028. 
[22] S. Bellucci, S. Krivonos, A. Marrani, E. Orazi, "Root" Action for N=4 Supersymmetric Mechanics Theories, Phys. Rev. D73 (2006) 025011, arXiv:hep-th/0511249

[23] F. Delduc, E. Ivanov, Gauging N=4 Supersymmetric Mechanics, Nucl. Phys. B753 (2006) 211, arXiv:hep-th/0605211[hep-th].

[24] S. Krivonos, A. Shcherbakov, N=4, $d=1$ tensor multiplet and hyper-Kahler sigma-models, Phys. Lett. B637 (2006) 119, arXiv:hep-th/0602113.

[25] S. Bellucci, S. Krivonos, O. Lechtenfeld, A. Shcherbakov, Superfield Formulation of Nonlinear N=4 Supermultiplets, Phys. Rev. D77 (2008) 045026, arXiv:0710.3832[hep-th].

[26] S. Bellucci, S. Krivonos, Geometry of $N=4, d=1$ nonlinear supermultiplet, Phys. Rev. D 74 (2006) 125024, arXiv:hep-th/0611104; S. Bellucci, S. Krivonos, V. Ohanyan, $N=4$ Supersymmetric MICZ-Kepler systems on $S^{3}$, Phys. Rev. D 76 (2007) 105023, arXiv:0706.1469[hep-th].

[27] E.A. Ivanov, S.O. Krivonos, V.M. Leviant, Geometric Superfield Approach To Superconformal Mechanics, J.Phys. A22 (1989) 4201.

[28] M. de Crombrugghe, V. Rittenberg, Supersymmetric Quantum Mechanics, Ann. Phys. 151 (1983) 99.

[29] E.A. Ivanov, A.V. Smilga, Supersymmetric gauge quantum mechanics: Superfield description, Phys. Lett. B257 (1991) 79;

V.P. Berezovoj, A.I. Pashnev, Three-dimensional $N=4$ extended supersymmetrical quantum mechanics, Class. Quant. Grav. 8 (1991) 2141;

A. Maloney, M. Spradlin, A. Strominger, Superconformal multiblack hole moduli spaces in fourdimensions, JHEP 0204 (2002) 003, arXiv:hep-th/9911001.

[30] T.T. Wu, C.N. Yang, Some solutions of the classical isotopic gauge field equations, in Properties of Matter under Unusual Conditions, Festschrift for the 60th birthday of E. Teller, p.349, Ed. H. Mark and S. Fernbach, Interscience: (1969);

T.T. Wu, C.N. Yang, Some Remarks About Unquantized Nonabelian Gauge Fields, Phys. Rev. D12 (1975) 3843.

[31] G.W. Gibbons, S.W. Hawking, Gravitational Multi - Instantons, Phys. Lett. B78 (1978) 430.

[32] C.N. Pope, A.L. Yuille, A Yang-Mills Instanton in Taub-NUT Space, Phys. Lett. 78B (1978) 424.

[33] G. Etesi, T. Hausel, On Yang-Mills instantons over mulyi-centered gravitational instantons, Comm. Math. Phys. 235 (2003) 275, arXiv:hep-th/0207196.

[34] S. Bellucci, S. Krivonos, A. Shcherbakov, Generic N=4 supersymmetric hyper-Kahler sigma models in $D=1$, Phys. Lett. B645 (2007) 299, arXiv:hep-th/0611248. 